\title{
Characterization and rate of symptomatic adjacent-segment disease after index lateral lumbar interbody fusion: a single-institution, multisurgeon case series with long-term follow-up
}

\author{
Timothy Y. Wang, MD, Vikram A. Mehta, MD, MPH, Eric W. Sankey, MD, Khoi D. Than, MD, \\ C. Rory Goodwin, MD, PhD, Isaac O. Karikari, MD, Robert E. Isaacs, MD, and \\ Muhammad M. Abd-El-Barr, MD, PhD
}

Department of Neurological Surgery, Duke University Medical Center, Durham, North Carolina

OBJECTIVE The rate of symptomatic adjacent-segment disease (ASD) after newer minimally invasive techniques, such as lateral lumbar interbody fusion (LLIF), is not known. This study aimed to assess the incidence of surgically significant ASD in adult patients who have undergone index LLIF and to identify any predictive factors.

METHODS Patients who underwent index LLIF with or without additional posterior pedicle screw fixation between 2010 and 2012 and received a minimum of 2 years of postoperative follow-up were retrospectively included. Demographic and perioperative data were recorded, as well as radiographic data and immediate perioperative complications. The primary endpoint was revision surgery at the level above or below the previous construct, from which a survivorship model of patients with surgically significant symptomatic ASD was created.

RESULTS Sixty-seven patients with a total of 163 interbody levels were included in this analysis. In total, 17 (25.4\%) patients developed surgically significant ASD and required additional surgery, with a mean \pm SD time to revision of 3.59 \pm 2.55 years. The mean annual rate of surgically significant ASD was $3.49 \%$ over 7.27 years, which was the average follow-up. One-third of patients developed significant disease within 2 years of index surgery, and 1 patient required surgery at the adjacent level within 1 year. Constructs spanning 3 or fewer interbody levels were significantly associated with increased risk of surgically significant ASD; however, instrument termination at the thoracolumbar junction did not increase this risk. Surgically significant ASD was not impacted by preoperative disc height, foraminal area at the adjacent levels, or changes in global or segmental lumbar lordosis.

CONCLUSIONS The risk of surgically significant ASD after LLIF was similar to the previously reported rates of other minimally invasive spine procedures. Patients with shorter constructs had higher rates of subsequent ASD.

https://thejns.org/doi/abs/10.3171/2020.10.SPINE201635

KEYWORDS adjacent-segment disease; revision; lateral lumbar interbody fusion; degenerative

$\mathrm{L}$ ATERAL lumbar interbody fusion (LLIF), first introduced in 2001 and later formalized by Pimenta and Ozgur in 2006, is a well-established lateral approach that allows for indirect decompression and fusion of the thoracolumbar spine. ${ }^{1}$ However, the fusion of two or more vertebrae with any method places additional biomechanical stress on the vertebral bodies and intervertebral discs above and below the construct. ${ }^{2}$ This frequently results in pathological, accelerated degenerative changes at those levels known as adjacent-segment degeneration when radiographically present but clinically silent or adjacent-segment disease (ASD) when radiographically present and clinically symptomatic. Patients with ASD develop new back pain or radiculopathies associated with the involved levels due to facet hypertrophy, disc degeneration, foraminal stenosis, and ligamentous hypertrophy that may ultimately require correction with additional thoracolumbar surgery. ${ }^{3}$ Surgically significant ASD contributes significantly to long-term morbidity and is associated with a large financial burden, with costs averaging $\$ 32,915$ but

ABBREVIATIONS ALIF = anterior lumbar interbody fusion; ASD = adjacent-segment disease; LLIF = lateral lumbar interbody fusion; TLIF = transforaminal lumbar interbody fusion.

SUBMITTED September 6, 2020. ACCEPTED October 12, 2020.

INCLUDE WHEN CITING Published online May 21, 2021; DOI: 10.3171/2020.10.SPINE201635. 
ranging as high as $\$ 63,769$ per repeat lumbar surgery. ${ }^{4}$ Thus, understanding the rate and clinical factors predictive of surgically significant ASD will aid in patient selection and operative planning, with the goals of reducing patient morbidity and financial costs while maximizing positive outcomes.

The rate of ASD after various lumbar spine surgical procedures has been well studied and varies widely depending on the type of fusion procedure and location within the spinal axis. In traditional posterior lumbar interbody fusion, rates of radiographic ASD at the 10year follow-up are as high as $75 \%,{ }^{5}$ with prevalence of surgically significant ASD varying from 6.3\% to $30 \% .^{6,7}$ Similar long-term prevalence rates of surgical ASD are reported for open and minimally invasive transforaminal lumbar interbody fusion (TLIF) $(9.2 \%-30 \%){ }^{8}{ }^{8}{ }^{9}$ Depending on study-specific definitions and criteria, large-cohort studies report prevalence rates of ASD that vary from $1.9 \%$ to $30 \%$ for as long as 10 years postoperatively in patients who have undergone lumbar fusion, all-inclusive of the various surgical approaches. For most minimally invasive approaches, the average prevalence rate is approximately $2 \%-3.5 \%$ per year. ${ }^{10,11}$

Unfortunately, there is a paucity of such data for patients who have undergone LLIF. Given these unknowns, this study attempts to describe the rate, timeline, and predictors of surgically significant ASD in patients who have undergone index LLIF.

\section{Methods \\ Study Design}

This academic, single-institution, multisurgeon retrospective review of patients who underwent thoracolumbar fusion via the LLIF approach was performed to identify rates and predictors of surgically significant ASD.

\section{Patient Selection}

We retrospectively included consecutive patients who underwent LLIF between January 1, 2010, and September 31, 2012, by four surgeons with board certification in either neurological surgery or orthopedic surgery and spine fellowship training. Inclusion criteria were as follows: 1) age 18 years or older; 2) at least 6 weeks of failed conservative management for low-back pain, neurogenic claudication, or lower-extremity radicular symptoms; 3) LLIF with or without posterior spinal fusion with pedicle screws; and 4) at least 2 years of follow-up. Posterior pedicle screw supplementation was performed for patients with spondylolisthesis or for patients undergoing LLIF for coronal or sagittal deformity correction; ultimately, the inclusion of posterior instrumentation was performed on a case-by-case basis.

Patients who had 1) previous thoracolumbar spine surgery or 2) surgery for neoplastic, traumatic, or infectious etiology were excluded. Patients who had undergone corpectomy with the lateral approach were also excluded, as well as patients who had pedicle screw instrumentation extending beyond their lateral fusion construct. To reduce the number of patients lost to follow-up, those who did not have at least 2 years of correspondence in our health re- cords were contacted via telephone to determine whether they had undergone additional surgery at the level above or below the original LLIF.

\section{Data Collection}

We recorded patient age, sex, BMI, relevant baseline medical comorbidities (hypertension, hyperlipidemia, diabetes mellitus, smoking history, rheumatoid arthritis, osteoporosis), LLIF interbody levels, posterior instrumentation levels, upper instrumented vertebra, involvement of the thoracolumbar junction (L1 or L2), immediate 30-day postoperative complications (infection, pulmonary embolism, myocardial infarction, death), additional unplanned surgery at index levels, and 30-day readmission.

Radiographic variables, including preoperative and postoperative lumbar lordosis, segmental lumbar lordosis, and cage height, were measured. Additionally, disc height and foraminal area at the levels adjacent to the index spinal levels were also recorded. For patients with ASD, changes in disc height and foraminal height were measured at the levels of the adjacent segments.

The primary outcome of this study was ASD resulting in additional thoracolumbar surgery. This included extension of fusion, decompression, vertebral column reconstruction, corpectomy, and osteotomy involving the upper or lower vertebral level. For patients who met these criteria, the time from index surgery to revision surgery was recorded, as was the involved level(s). Regardless of the interval between index and revision surgery, any surgery involving the adjacent levels, not including hematoma evacuation or infection washout, was considered as evidence of surgically significant ASD. Institutional review board approval was obtained prior to data collection, and patient consent was not required unless patients were contacted via telephone.

\section{Statistical Analysis}

The primary aim of this study was to determine the incidence of surgically significant ASD. The secondary aim was to determine the rate of survivorship until the development of surgically significant ASD, as well as to identify clinical or surgical predictive factors significantly associated with increased risk of ASD. Parametric data were expressed as means \pm SD and compared with the Fisher exact test. Categorical data were compared with the chi-square test, with $\alpha<0.05$ indicating statistical significance.

\section{Results}

A total of 165 patients underwent LLIF over the study period. Of these, 19 (11.5\%) were excluded due to a lack of 2-year follow-up data. Sixty-nine (41.8\%) patients had undergone previous thoracolumbar spine surgery and were excluded. Ten additional patients were excluded because they had surgical hardware extending beyond the interbody spinal levels. We included the remaining 67 (40.6\%) eligible patients in the analysis (42 [61.8\%] women; mean \pm SD age $65.0 \pm 11.6$ years; mean BMI $28.1 \pm 5.8 \mathrm{~kg} / \mathrm{m}^{2}$ ) (Fig. 1). There were high incidence rates of preoperative hypertension $(n=32[47.8 \%])$ and hyperlipidemia $(n=29$ [43.3\%]) in this cohort, but low rates of diabetes $(\mathrm{n}=5$ 
[7.5\%]) and osteoporosis $(\mathrm{n}=7$ [10.5\%]). Twelve (17.9\%) patients were active smokers. A total of 163 LLIF interbody cages were placed, of which 127 were supplemented with posterior instrumentation. Fusion constructs terminated at the thoracolumbar junction (L1 or L2) in 23 (34.3\%) patients. The mean (range) length of follow-up was $7.27(2.1-10.3)$ years.

A total of $17(25.4 \%)$ patients underwent revision thoracolumbar surgery that included the level above and/or below the previous LLIF surgical construct. Most revision procedures occurred at the L5-S1 level $(n=9$ [52.9\%]). Of the 17 additional surgical procedures, there were 4 (23.5\%) TLIF procedures and 4 anterior lumbar interbody fusion (ALIF) procedures at L5-S1. The remainder of the revision procedures comprised revision LLIF at adjacent levels $(n=7[41.2 \%])$, Smith-Petersen osteotomy, or discectomy. A case example is shown in Fig. 2.

The mean \pm SD time to revision was $3.59 \pm 2.55$ years (median 2.38 years), with the first revision occurring 0.56 years after index surgery and the last revision occurring as late as 8.62 years after index surgery. When we assessed survivorship, the average annual rate of surgically significant ASD was 3.49\% through 7.27 years, which was the average length of follow-up; however, approximately onethird $(n=6[35.3 \%])$ of patients who eventually developed surgically significant ASD underwent revision surgery within the first 24 months of index surgery. The survivorship model is summarized in Fig. 3, and annual incidence is demonstrated in Table 1. Eight (47.1\%) patients had failure at the level below their construct, 7 (41.2\%) had failure at the level above their construct, and $2(11.8 \%)$ had failure at levels both above and below their construct.

The vast majority of patients in our sample cohort (n $=53[79.1 \%]$ ) had constructs that ended at L5. Of these, $50(74.6 \%)$ patients had short interbody constructs consisting of 1,2, or 3 LLIF levels, and 17 (25.4\%) had long interbody constructs consisting of 4 or more LLIF levels. Nine $(18.0 \%)$ patients with short constructs ending at L5 had symptomatic ASD, but 1 (5.9\%) patient with long constructs ending at L5 had symptomatic ASD (OR 2.0, 95\% CI $0.54-7.71 ; \mathrm{p}=0.35)$. There were $17(25.4 \%)$ patients with 4 or more LLIF levels, and of these, only $1(5.9 \%)$ had symptomatic ASD. Of the patients who underwent posterior instrumentation, only 1 patient had screws placed via an open approach.

Univariate analysis did not demonstrate any statistically significant associations between patients who required revision surgery for symptomatic ASD and those who did not in terms of demographic characteristics, BMI, comorbidities, smoking history, posterior instrumentation levels, upper instrumented vertebra, and termination of the construct at the thoracolumbar junction $(\mathrm{p}>0.05)$.

Patients with constructs consisting of 3 levels or fewer were statistically more likely to have symptomatic ASD (p $=0.02$ ). Additionally, there were no differences in preoperative $\left(29.3^{\circ} \pm 11.1^{\circ}\right.$ vs $\left.34.7^{\circ} \pm 11.9^{\circ}, \mathrm{p}=0.11\right)$ and postoperative $\left(33.8^{\circ} \pm 9.3^{\circ}\right.$ vs $\left.38.7^{\circ} \pm 10.9^{\circ}, \mathrm{p}=0.11\right)$ lordosis between patients with ASD and those without ASD. Changes in lordosis between patients with ASD and those without were also similar $\left(4.3^{\circ} \pm 5.9^{\circ}\right.$ vs $\left.5.4^{\circ} \pm 0.8^{\circ}, \mathrm{p}=0.85\right)$, as were changes in segmental lumbar lordosis $\left(4.3^{\circ} \pm 6.1^{\circ}\right.$ vs

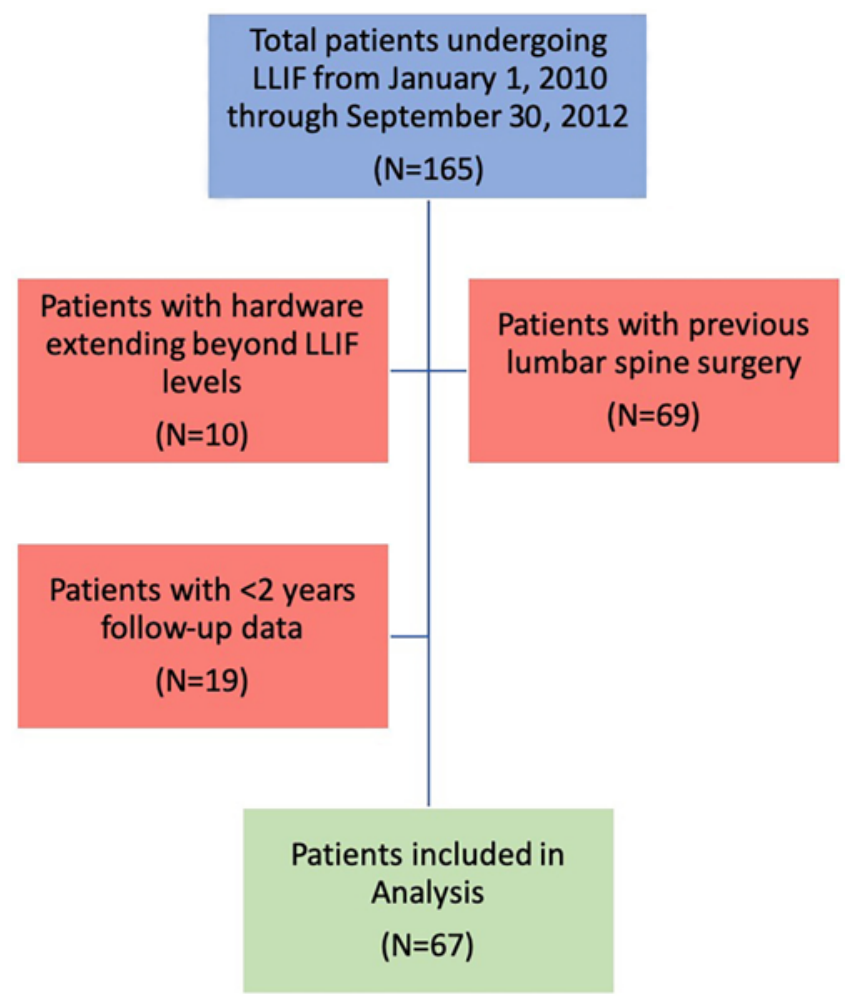

FIG. 1. Patient selection process. Figure is available in color online only.

$\left.3.2^{\circ} \pm 4.4^{\circ}, \mathrm{p}=0.43\right)$. Additionally, disc height $(8.5 \pm 3.3$ $\mathrm{mm}$ vs $9.5 \pm 2.3 \mathrm{~mm}, \mathrm{p}=0.21)$ and foraminal area $(1.5$ $\pm 2.4 \mathrm{~cm}^{2}$ vs $\left.0.9 \pm 0.3 \mathrm{~cm}^{2}, \mathrm{p}=0.12\right)$ at the adjacent levels were not correlated with increased risk of ASD. Cage height did not differ significantly between patients without ASD and those with surgically significant ASD $(9.6 \pm 1.3$ $\mathrm{mm}$ vs $10.0 \pm 1.9 \mathrm{~mm}, \mathrm{p}=0.52$ ). For patients with surgically significant ASD, the mean \pm SD loss of disc height at the affected level was $2.1 \pm 1.9 \mathrm{~mm}$ or $18.1 \% \pm 11.5 \%$, and the mean \pm SD reduction of the foraminal area was $0.19 \pm 0.10 \mathrm{~cm}^{3}$.

Rates of postoperative infection, myocardial infarction, pulmonary embolism, and deep vein thrombosis were all statistically similar between groups $(\mathrm{p}>0.05)$ (Tables 2 and 3). Additional surgical procedures and the involved levels are summarized in Table 4. Of all 67 patients, posterior instrumentation was performed in $55(82.1 \%)$. Of the 17 patients who developed ASD, 15 (88.2\%) had posterior instrumentation, whereas 39 of the remaining 50 (78.0\%) patients without surgically significant ASD had posterior instrumentation $(\mathrm{p}=0.36)$. Of the patients with ASD and posterior instrumentation, 8 patients had revision surgery involving the superior vertebral level; of these, all $8 \mathrm{had}$ posterior instrumentation. After reviewing the postoperative CT and MRI scans of all 8 patients, we determined that only $1(12.5 \%)$ of these patients had rostral facet violation attributable to the most rostral pedicle screw (Fig. 4).

\section{Discussion}

Spinal fusion surgery can accelerate the rate of radio- 

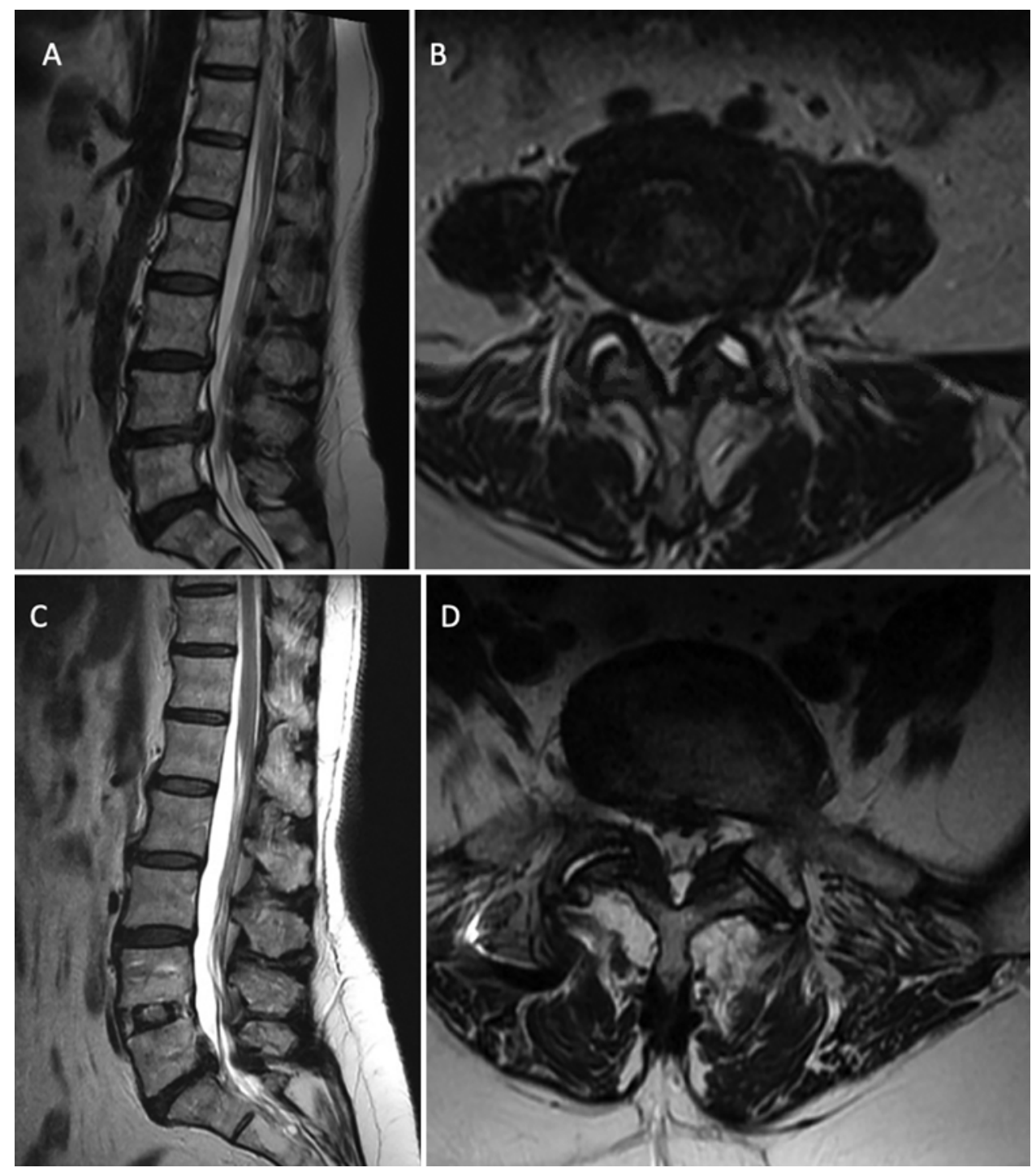

FIG. 2. Preoperative sagittal (A) and axial (B) T2-weighted lumbar spine MR images obtained in a patient who underwent L4-5 LLIF with posterior pedicle screw instrumentation. Postoperative sagittal (C) and axial (L5-S1) (D) T2-weighted lumbar spine MR images obtained at the 2-year follow-up. The patient developed worsening outer thigh and outer calf pain, consistent with L5-S1 ASD. The patient subsequently underwent L5-S1 TLIF, with resolution of symptoms.

graphic spondyloarthropic changes at the vertebral levels above and below the fusion construct; this phenomenon is termed adjacent-segment degeneration. A wide range of rates is quoted for adjacent-segment degeneration in patients who have undergone lumbar spine surgery; this is due in large part to the nonstandardized definition of adjacent-segment degeneration. Instead, the rate of symptomatic adjacent-segment degeneration is more commonly referenced, because these patients have debilitating back pain, radiculopathy, or deformity associated with the affected levels. ASD has significant impacts on quality of life and healthcare costs, especially for those patients who require additional surgery for treatment of this pathology.

This study demonstrates a cumulative prevalence rate of $25.4 \%$ and a mean annual incidence rate of $3.49 \%$ for surgically significant ASD through an average follow-up of 7.27 years in a patient population that received a mini- mum of 2 years of follow-up. These rates are similar to those of traditional open or more modern minimally invasive approaches.

When treating patients with thoracolumbar disease, surgeons must weigh approach (e.g., minimally invasive vs open, posterior vs lateral vs anterior), as well as which levels to treat. These decisions impact blood loss, length of hospital stay, and risks of morbidity and other complications. The number of levels fused and the upper instrumented vertebra, for example, are particularly important in spinal surgery. The general consensus recommends against inclusion of the apex of a deformity in the upper instrumented vertebra and against termination of constructs at the thoracolumbar junction. ${ }^{12}$ LLIF is increasingly used to treat deformity, but until now it has been unclear whether the same deformity concepts apply to this approach. This study demonstrates that patients with fu- 


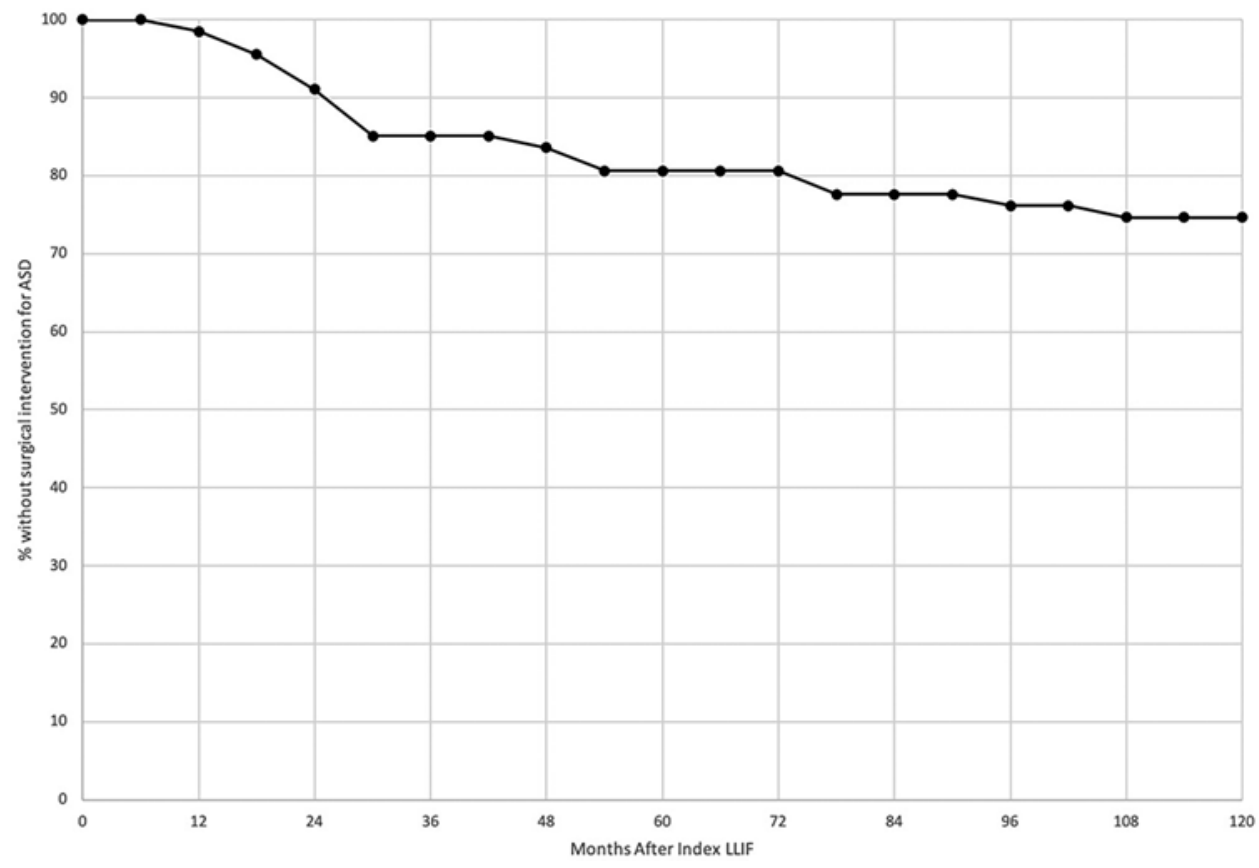

FIG. 3. Survivorship model of surgically significant ASD over time.

sion constructs terminating at the thoracolumbar junction did not have a statistically higher rate of ASD. Additionally, it appears that patients with ASD did not have significantly different postoperative lumbar lordosis or change in lumbar lordosis compared with those without ASD. This is contrary to some reports in the literature that suggest patients with lumbar ASD are more likely to experience overcorrection or undercorrection of lumbar lordosis. ${ }^{13,14}$

Additionally, long-segment constructs, which typically increase lever-arm and biomechanical stress at adjacent levels compared with shorter constructs, were not associated with increased rates of ASD. Even long constructs consisting of more than 4 levels had very low risk of ASD, which refutes previous claims to the contrary. This holds true even if L5 is the lower instrumented vertebra of the construct. Although the rate of ASD at L5 was not statistically different between patients with short constructs and those with long constructs, L5 is still the most common area for the development of ASD. This suggests the need to consider simultaneous treatment of any existing L5-S1 pathology in patients who are planning to undergo shortsegment LLIF with instrument termination at L5. Unfortunately, one of the inherent drawbacks of LLIF is the inability to access the L5-S1 disc space due to obstruction by the iliac crest.

Newer lateral antepsoas techniques, such as oblique lateral interbody fusion, had not been developed during the period defined by this study. Today, these techniques should be considered for the subset of patients with evident L5-S1 disease, as the surgeon can address all disc spaces from a single lateral position. Whether this would decrease the incidence of ASD is currently unknown but worth investigation. However, our results suggest that there were no significant differences in foraminal or disc pathology at the adjacent levels between patients with ASD and those without ASD. This suggests that the underlying pathology at the adjacent levels was not simply ignored as part of the original operative plan.

There are additional technique-specific considerations regarding the development of ASD in patients who undergo index LLIF. In this series, the vast majority of LLIF cases were supplemented with percutaneous pedicle screw fixation. Placement of percutaneous pedicle screws carries increased risk of facet violation, which places the adjacent segments at higher risk for destabilization compared with screws placed with open posterior techniques. ${ }^{15,16}$ That being said, in our cohort, there were no significant differences in surgically significant ASD between patients who underwent stand-alone LLIF and those who underwent

TABLE 1. Annual incidence of ASD

\begin{tabular}{ccccc}
\hline \multirow{2}{*}{$\begin{array}{c}\text { Follow-Up, } \\
\text { Yrs }\end{array}$} & Included & $\begin{array}{c}\text { Lost to } \\
\text { Follow-Up }\end{array}$ & $\begin{array}{c}\text { w/ New } \\
\text { ASD }\end{array}$ & $\begin{array}{c}\text { Annual Incidence } \\
\text { of ASD, \% }\end{array}$ \\
\hline 1 & 67 & 0 & 1 & 1.49 \\
\hline 2 & 67 & 0 & 5 & 7.46 \\
\hline 3 & 59 & 8 & 4 & 6.78 \\
\hline 4 & 55 & 4 & 1 & 1.82 \\
\hline 5 & 51 & 4 & 2 & 3.92 \\
\hline 6 & 49 & 2 & 0 & 0.00 \\
\hline 7 & 45 & 4 & 2 & 4.44 \\
\hline 8 & 41 & 4 & 1 & 2.44 \\
\hline 9 & 15 & 26 & 1 & 6.67 \\
\hline 10 & 4 & 11 & 0 & 0.00 \\
\hline 11 & 0 & 4 & 0 & 0.00 \\
\hline
\end{tabular}


TABLE 2. Patient characteristics

\begin{tabular}{|c|c|c|c|c|}
\hline Characteristic & No Surgical ASD & Surgical ASD & Total & $\mathrm{p}$ Value \\
\hline No. of patients & 50 & 17 & 67 & NA \\
\hline \multicolumn{5}{|l|}{ Demographic characteristics } \\
\hline Age, yrs & $65.7 \pm 12.3$ & $63.3 \pm 9.0$ & $65.0 \pm 11.6$ & 0.23 \\
\hline Male & $21(42.0)$ & $4(23.5)$ & $25(37.3)$ & 0.25 \\
\hline $\mathrm{BMI}$ & $28.1 \pm 0.8$ & $28.1 \pm 1.4$ & $28.1 \pm 5.8$ & 0.98 \\
\hline \multicolumn{5}{|l|}{ Comorbidity } \\
\hline Hypertension & $27(54.0)$ & $10(58.8)$ & $37(55.2)$ & 0.78 \\
\hline Hyperlipidemia & $25(50.0)$ & $9(52.9)$ & $34(50.7)$ & $>0.99$ \\
\hline Diabetes mellitus & $4(8.0)$ & $4(23.5)$ & $8(11.9)$ & 0.21 \\
\hline Osteoporosis & $7(14.0)$ & $0(0)$ & $7(10.4)$ & 0.18 \\
\hline Smoking history & $11(22.0)$ & $1(5.9)$ & $12(17.9)$ & 0.27 \\
\hline \multicolumn{5}{|l|}{ LLIF level } \\
\hline T8-9 & $0(0)$ & $0(0)$ & $0(0)$ & NA \\
\hline T9-10 & $0(0)$ & $0(0)$ & $0(0)$ & NA \\
\hline T10-11 & $2(4.0)$ & $0(0)$ & $2(3.0)$ & $>0.99$ \\
\hline $\mathrm{T} 11-12$ & $5(10.0)$ & $0(0)$ & $5(7.5)$ & 0.58 \\
\hline T12-L1 & $9(50.0)$ & $1(5.9)$ & $10(14.9)$ & 0.43 \\
\hline L1-2 & $16(32.0)$ & $2(11.8)$ & $18(26.9)$ & 0.13 \\
\hline L2-3 & $25(50.0)$ & $7(41.2)$ & $32(47.8)$ & 0.58 \\
\hline L3-4 & $30(60.0)$ & $8(47.1)$ & $38(56.7)$ & 0.40 \\
\hline L4-5 & $45(90.0)$ & $13(76.5)$ & $58(86.6)$ & 0.22 \\
\hline \multicolumn{5}{|l|}{ Posterior screw level } \\
\hline T8-9 & $0(0)$ & $0(0)$ & $0(0)$ & NA \\
\hline T9-10 & $0(0)$ & $0(0)$ & $0(0)$ & NA \\
\hline T10-11 & $2(4.0)$ & $0(0)$ & $2(3.0)$ & $>0.99$ \\
\hline T11-12 & $5(10.0)$ & $0(0)$ & $5(7.5)$ & 0.32 \\
\hline T12-L1 & $9(18.0)$ & $1(5.9)$ & $10(14.9)$ & 0.43 \\
\hline L1-2 & $12(24.0)$ & $1(5.9)$ & $13(19.4)$ & 0.16 \\
\hline L2-3 & $18(36.0)$ & $5(29.4)$ & $23(34.3)$ & 0.77 \\
\hline L3-4 & $22(44.0)$ & $6(35.3)$ & $28(41.8)$ & 0.58 \\
\hline L4-5 & $35(70.0)$ & $11(64.7)$ & $46(68.7)$ & 0.77 \\
\hline Termination at thoracolumbar junction & $16(32.0)$ & $7(41.2)$ & $23(34.3)$ & 0.56 \\
\hline$>3$ total instrumented levels & $16(32.0)$ & $1(5.9)$ & $17(25.4)$ & 0.03 \\
\hline
\end{tabular}

$\mathrm{NA}=$ not applicable

Values are shown as number (percentage) or mean \pm SD. Boldface type indicates statistical significance.

TABLE 3. Postoperative complications after index surgery

\begin{tabular}{lcccc}
\hline \multicolumn{1}{c}{ Complication } & No Surgical ASD & Surgical ASD & Total & $p$ Value \\
\hline Infection & $2(4.0)$ & $0(0)$ & $2(3.0)$ & $>0.99$ \\
\hline Pulmonary embolism & $0(0)$ & $1(5.9)$ & $1(1.5)$ & $>0.99$ \\
\hline Deep vein thrombosis & $0(0)$ & $0(0)$ & $0(0)$ & $\mathrm{NA}$ \\
\hline Myocardial infarction & $0(0)$ & $0(0)$ & $0(0)$ & $\mathrm{NA}$ \\
\hline Unplanned additional surgery at index level & $3(6.0)$ & $0(0)$ & $3(4.5)$ & $>0.99$ \\
\hline 30-day readmission & $0(0)$ & $0(0)$ & $0(0)$ & $\mathrm{NA}$ \\
\hline
\end{tabular}

Values are shown as number (percentage). 
TABLE 4. Additional surgical procedures for ASD

\begin{tabular}{cc}
\hline \multicolumn{1}{c}{ Characteristic } & Value \\
\hline Procedure, $\mathrm{n}$ & \\
\hline ALIF & 4 \\
\hline Smith-Petersen osteotomy & 1 \\
\hline TLIF & 4 \\
\hline LLIF & 7 \\
\hline Discectomy & 1 \\
\hline Total & 17 \\
\hline Revised level, $\mathrm{n}$ & 2 \\
\hline T12-L1 & 2 \\
\hline L1-2 & 1 \\
\hline L2-3 & 3 \\
\hline L3-4 & 0 \\
\hline L4-5 & 9 \\
\hline L5-S1 & $3.59 \pm 2.55$ \\
\hline Time to revision, yrs & 2.38 \\
\hline Mean \pm SD, yrs & $0.56-8.62$ \\
\hline Median &
\end{tabular}

LLIF with posterior screw fixation, and the rate of facet joint violation was low; however, this could have been limited by the relatively small patient population.

This study has limitations. First, as a retrospective analysis, we relied on medical records to provide data about follow-up and need for additional surgery at adjacent levels. Naturally, a subset of patients who underwent index LLIF did not receive the minimum 2 years of follow-up and were thus excluded from the study. Although this is purely speculative, it is also possible that these patients did not seek follow-up because they were asymptomatic after surgery. We attempted to minimize this bias by recontacting all patients in this cohort, but naturally there were patients who were truly lost to follow-up. However, this is the first study to have extended follow-up for patients undergoing LLIF, with some patients receiving follow-up through 10 years. Additionally, the clinical threshold at which patients are offered additional surgery for ASD varies widely among surgeons. Although this study attempted to eliminate this confounding factor by including multiple surgeons, it is important to recognize that the lack of a standardized threshold at which ASD warrants surgery may cause variations in the reported rates and timing of revision, depending on the practice preferences of the surgeon.

Patient-reported outcomes were also not collected for this cohort. These would be useful for interpreting patients' short- and long-term perceptions of quality of life and symptomatology and will be of critical importance for forthcoming prospective studies. Additionally, the patients in this study were included during a period when full-length scoliosis radiographs were not considered standard of care. As a result, the medical records did not have complete access to full-length scoliosis radiographs of the included patients, which reduced our ability to assess the impact of spinopelvic parameters on the incidence of sur-

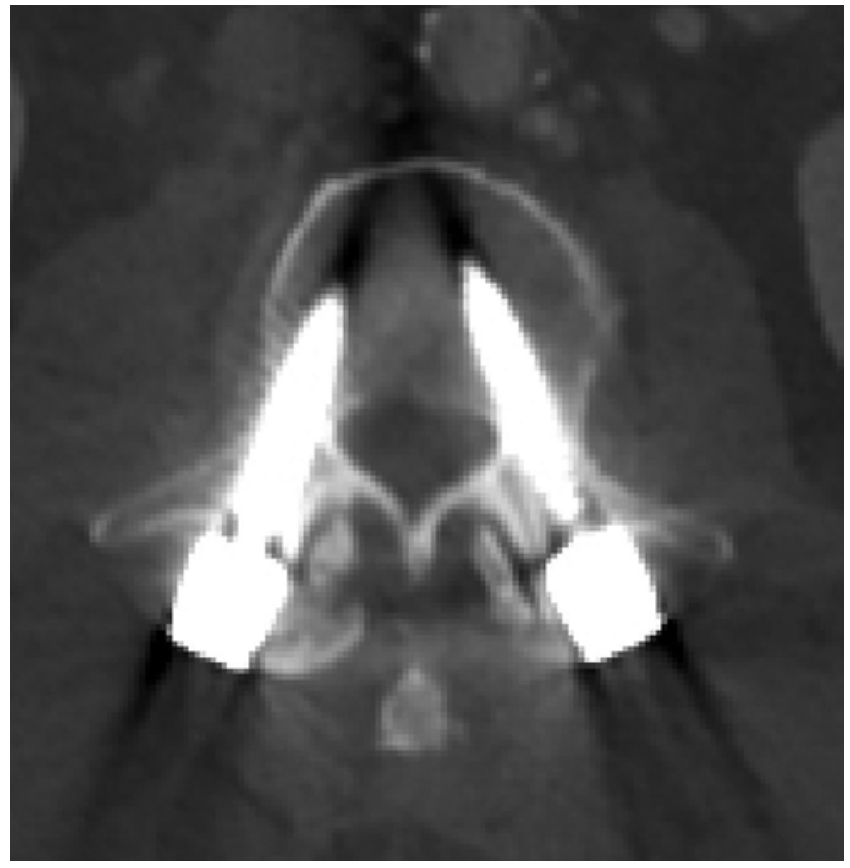

FIG. 4. Image obtained from a patient who previously underwent $L 4-5$ LLIF with damage to the right L3-4 facet by the right-side L 4 screw. The entry point of the screw is slightly medial and results in a small inferior articulating process fracture at the right $\mathrm{L} 3$.

gically significant ASD. The inclusion of patients from this period, however, allowed for significantly longer followup, which is a significant strength of this study that has not been replicated by any other studies on LLIF.

These spinopelvic factors are increasingly well-recognized influencers of ASD but were not as well understood at the time that the included patients underwent surgery. With improved understanding of the wide impact and clinical significance of spinopelvic parameters, preoperative and postoperative full-length scoliosis radiographs are obtained for all patients considering thoracolumbar spine surgery at our institution. Additional forthcoming research on this topic will certainly include these data.

Nonetheless, this is the first study to quantify the rate of surgically significant ASD after index LLIF for patients with at least 2 years of follow-up. This is also the first study to suggest that long LLIF constructs, or constructs that terminate at the thoracolumbar junction, are not associated with significantly increased rates of ASD relative to other lumbar fusion surgical procedures. Furthermore, this study suggests that short constructs, especially those that terminate at L5, place patients at significantly higher risk for ASD. These patients can potentially undergo oblique or anterior-to-psoas techniques at L5-S1, which were unavailable during the study period (2010-2012), or same-day alternate-position surgery (e.g., TLIF-posterior, ALIF-anterior) to address this level. Additional large, multicenter, prospective studies with standardized preoperative and postoperative scoliosis imaging, as well as comprehensive follow-up, are needed to better characterize ASD after LLIF. 


\section{Conclusions}

We found that patients who underwent LLIF had a rate of surgically significant ASD that is similar to the rates of more established open and minimally invasive approaches for thoracolumbar spine fusion. Although this study did not demonstrate any significant demographic predictors of increased risk of surgically significant ASD, it does suggest that long constructs and termination of constructs at the thoracolumbar junction do not increase the risk of ASD. Additionally, patients with disease at L3-5, who would otherwise require short LLIF constructs with L5 as the lower instrumented vertebra, should consider fusion that includes the L5-S1 disc space. To date, this is the first and largest series to specifically address the rate of surgically significant ASD in patients who have undergone index LLIF.

\section{References}

1. Ozgur BM, Aryan HE, Pimenta L, Taylor WR. Extreme Lateral Interbody Fusion (XLIF): a novel surgical technique for anterior lumbar interbody fusion. Spine J. 2006;6(4):435-443.

2. Kelly BP, Theodore N. What can standard in vitro biomechanical testing tell us about adjacent segment disease? World Neurosurg. 2016;89:710-712.

3. Virk SS, Niedermeier S, Yu E, Khan SN. Adjacent segment disease. Orthopedics. 2014;37(8):547-555.

4. Parker SL, Shau DN, Mendenhall SK, McGirt MJ. Factors influencing 2-year health care costs in patients undergoing revision lumbar fusion procedures. J Neurosurg Spine. 2012; 16(4):323-328.

5. Okuda S, Nagamoto Y, Matsumoto T, et al. Adjacent segment disease after single segment posterior lumbar interbody fusion for degenerative spondylolisthesis: minimum 10 years follow-up. Spine (Phila Pa 1976). 2018;43(23):E1384-E1388.

6. Okuda S, Yamashita T, Matsumoto T, et al. Adjacent segment disease after posterior lumbar interbody fusion: a case series of 1000 patients. Global Spine J. 2018;8(7):722-727.

7. Wang H, Ma L, Yang D, et al. Incidence and risk factors of adjacent segment disease following posterior decompression and instrumented fusion for degenerative lumbar disorders. Medicine (Baltimore). 2017;96(5):e6032.

8. Yamasaki K, Hoshino M, Omori K, et al. Risk factors of adjacent segment disease after transforaminal inter-body fusion for degenerative lumbar disease. Spine (Phila Pa 1976). 2017; 42(2):E86-E92.

9. Yee TJ, Terman SW, La Marca F, Park P. Comparison of adjacent segment disease after minimally invasive or open transforaminal lumbar interbody fusion. J Clin Neurosci. 2014;21(10):1796-1801.

10. Bae JS, Lee SH, Kim JS, et al. Adjacent segment degeneration after lumbar interbody fusion with percutaneous pedicle screw fixation for adult low-grade isthmic spondylolisthesis: minimum 3 years of follow-up. Neurosurgery. 2010;67(6): 1600-1608.
11. Epstein NE. Adjacent level disease following lumbar spine surgery: a review. Surg Neurol Int. 2015;6(24)(suppl 24): S591-S599.

12. Park SJ, Lee CS, Chung SS, et al. different risk factors of proximal junctional kyphosis and proximal junctional failure following long instrumented fusion to the sacrum for adult spinal deformity: survivorship analysis of 160 patients. Neurosurgery. 2017;80(2):279-286.

13. Zheng G, Wang C, Wang T, et al. Relationship between postoperative lordosis distribution index and adjacent segment disease following L4-S1 posterior lumbar interbody fusion. $J$ Orthop Surg Res. 2020;15(1):129.

14. Wang SJ, Zhang SB, Yi YY, et al. Estimation of the ideal correction of lumbar lordosis to prevent reoperation for symptomatic adjacent segment disease after lumbar fusion in older people. BMC Musculoskelet Disord. 2020;21(1):429.

15. Babu R, Park JG, Mehta AI, et al. Comparison of superiorlevel facet joint violations during open and percutaneous pedicle screw placement. Neurosurgery. 2012;71(5):962-970.

16. Patel RD, Graziano GP, Vanderhave KL, et al. Facet violation with the placement of percutaneous pedicle screws. Spine (Phila Pa 1976). 2011;36(26):E1749-E1752.

\section{Disclosures}

Dr. Goodwin is supported by grants from the NIH/NINDS K12 NRCDP Physician Scientist Award (2K12NS080223-06) and the Robert Wood Johnson Harold Amos Medical Faculty Grant. Dr. Karikari is a consultant for NuVasive, receives fellowship funding from NuVasive, and is a member of the advisory board for the Johnson \& Johnson Adult Deformity Group. Dr. Than is a consultant for Bioventus and receives honoraria from LifeNet Health and DJO Development Program (RWJ 76238). Dr. Isaacs is a consultant for NuVasive and owns stock in TrackX. Dr. Abd-El-Barr is a consultant for Spineology.

\section{Author Contributions}

Conception and design: Wang, Isaacs. Acquisition of data: Wang, Isaacs, Abd-El-Barr. Analysis and interpretation of data: Wang. Drafting the article: Wang, Mehta. Critically revising the article: all authors. Reviewed submitted version of manuscript: Wang, Sankey, Than, Goodwin, Karikari, Isaacs, Abd-El-Barr. Approved the final version of the manuscript on behalf of all authors: Wang. Statistical analysis: Wang. Administrative/technical/material support: Isaacs, Abd-El-Barr. Study supervision: Isaacs, Abd-El-Barr.

\section{Supplemental Information}

Previous Presentations

Portions of this study were presented at the 2019 AANS Annual Scientific Meeting, San Diego, California, April 13-17, 2019.

\section{Correspondence}

Timothy Y. Wang: Duke University Medical Center, Durham, NC. timothy.wang@duke.edu. 